\title{
Estudo Termodinâmico e Cinético da Formação de Modelos Químicos Derivados de Adamantano para a Interação com Ciclodextrinas
}

\author{
Selma F. Bazan, Clara H. S. Braga, Frederico B. de Sousa \& Juliana F. Lopes
}

\section{Introdução}

Complexos de inclusão são objetos de estudos da química supramolecular, sendo que uma das moléculas mais estudadas para criar estes sistemas são as ciclodextrinas (CDs). A partir da formação de um composto de inclusão é possível, por exemplo, modular a liberação de um fármaco. ${ }^{1}$ Também é possível aperfeiçoar a eficiência e diminuir os efeitos colaterais já apresentados pelo fármaco, quando incluídos na cavidade das $\mathrm{CDs}^{2}{ }^{2} \mathrm{O}$ adamantano (Ad) é uma molécula que quando interage com ciclodextrinas, produz complexos de estabilidade alta, ${ }^{3}$ isso faz com que seja possível modificar a cadeia lateral desta molécula e estudar a estabilidade de diferentes complexos de inclusão modelos. ${ }^{2}$ Diante disso, pretendia-se realizar os estudos termodinâmicos de equilíbrio entre os complexos de inclusão, formados por ciclodextrinas e os modelos de adamantano, porém a dificuldade experimental na síntese de tais derivados de adamantano exigiu a mudança no foco do objetivo inicial. As reações a serem estudadas seguem na Figura 1 e 2 :

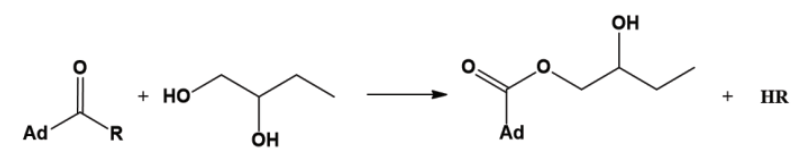

Figura 1. Primeira reação de esterificação $(\mathrm{R}=\mathrm{OH}$ ou $\mathrm{Cl})$

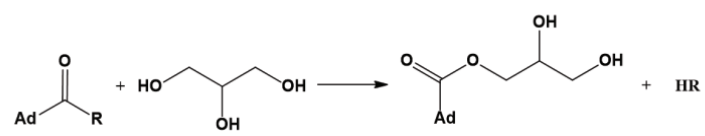

Figura 2. Primeira reação de esterificação $(\mathrm{R}=\mathrm{OH}$ ou $\mathrm{Cl})$

Os cálculos com a reação do Modelo $1,(\mathrm{R}=\mathrm{OH})$ apresentaram resultados termodinâmicos e cinéticos insatisfatórios $(\Delta \mathrm{G}>0$ ou próximo de 0 e $\Delta \mathrm{G}+>50 \mathrm{kcal} /$ $\mathrm{mol}$ ), o que foi observado experimentalmente, mesmo na presença de catalisadores como as carbodiimidas, esses resultados já foram apresentados em outro trabalho. Assim, os resultados que serão apresentados neste trabalho referem-se apenas ao estudo termodinâmico e 
cinético das reações do Modelo $2(\mathrm{R}=\mathrm{Cl})$, que tem como produtos as espécies a serem incluídas na cavidade da ciclodextrina.

\section{Metodologia}

Todos os cálculos foram realizados utilizando o programa Gaussian 09. Realizando otimização de geometria e análise vibracional com o nível de teoria DFT (M062x), funções de base 6-31g(d,p) para todos os átomos. Realizaram-se cálculos com o efeito do solvente utilizando o modelo contínuo (SMD). A obtenção dos derivados de Ad como descrito na Figura 1 foi realizada de acordo com metodologia adaptada da literatura, pela reação do derivado ácido do Ad com cloreto de tionila, para formação cloreto de do Ad e posterior reação em solvente anidro $\left(\mathrm{CH}_{2} \mathrm{C}_{12}\right)$ com o álcool da para obtenção do éster, Figura $1 .{ }^{4}$

\section{Resultados e Discussão}

Com o objetivo de estudar a estabilidade termodinâmica e cinética das reações em questão, cálculos foram realizados (reação Figura 1) para reagentes e produtos intermediários e isolados, como mostrado na Figura 3:
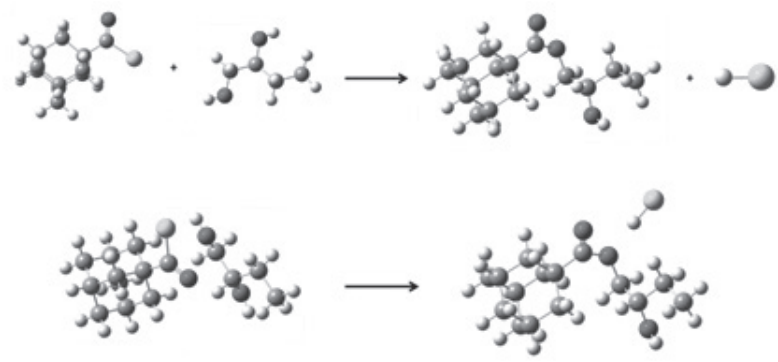

Figura 3. Estruturas otimizadas dos reagentes e produtos isolados e intermediários.

Também foi possível encontrar o estado de transição com o efeito do solvente da primeira reação. Sua estrutura apresenta-se na Figura 4 e a respectiva coordenada intrínseca da reação (IRC) é apresentada na Figura 5:

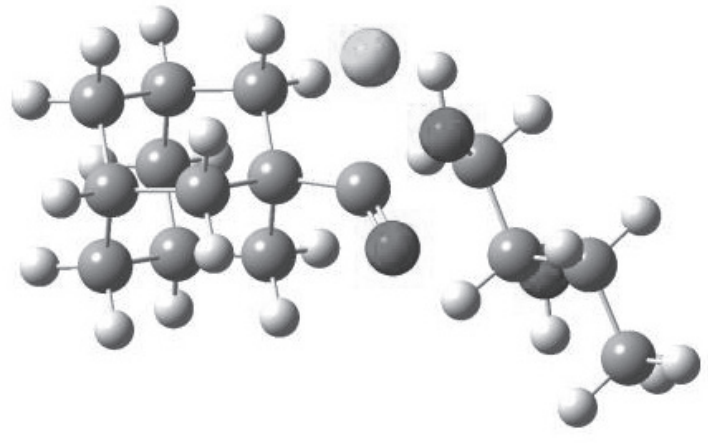

Figura 4. Estado de transição da primeira reação (M062x/SMD/196,12i)

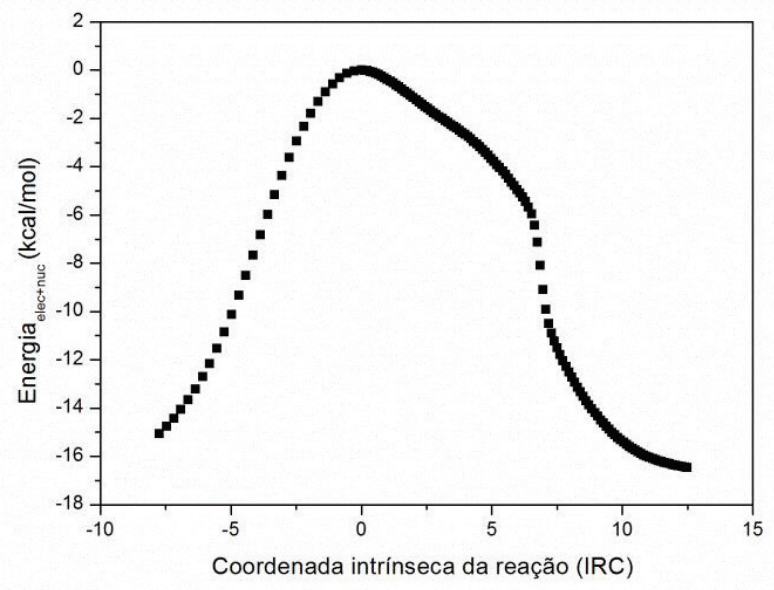

Figura 5. Gráfico da coordenada intrínseca da reação (IRC) para a primeira reação

Os cálculos foram iniciados com o efeito do solvente (água), pois as tentativas em fase gás não possibilitaram caracterizar o estado de transição. Porém após obter a estrutura em solução aquosa, os cálculos em fase gás foram feitos, tomando como a base a estrutura do estado de transição com solvente, assim como com o solvente utilizado na parte experimental (diclorometano).

Na Tabela 1 apresentam-se os resultados termodinâmicos e cinéticos obtidos: 
Tabela 1. Dados termodinâmicos e cinéticos dos cálculos descritos anteriormente $(\mathrm{kcal} / \mathrm{mol})$ :

\begin{tabular}{|c|c|c|c|c|}
\hline & $\Delta \mathrm{G}$ & $\Delta \mathrm{H}$ & $\mathrm{T} \Delta \mathrm{S}$ & $\Delta \mathrm{G}+$ \\
\hline $\mathrm{FG}-$ isolados & $-1,16$ & $-3,55$ & $-2,38$ & 29,70 \\
\hline $\begin{array}{c}\mathrm{FG}- \\
\text { intermediários }\end{array}$ & $-2,32$ & $-3,44$ & $-1,12$ & 27,27 \\
\hline $\begin{array}{c}\text { SMD (H2O) - } \\
\text { isolados }\end{array}$ & $-1,38$ & $-4,10$ & $-2,71$ & 25,59 \\
\hline $\begin{array}{c}\text { SMD (H2O) - } \\
\text { intermediários }\end{array}$ & $-4,10$ & $-3,95$ & 0,16 & 19,67 \\
\hline $\begin{array}{c}\text { SMD (CH2Cl2) - } \\
\text { Isolados }\end{array}$ & $-2,43$ & $-3,66$ & $-1,22$ & 24,16 \\
\hline $\begin{array}{c}\text { SMD (CH2Cl2) - } \\
\text { Intermediários }\end{array}$ & $-2,70$ & $-2,68$ & 0,02 & 21,56 \\
\hline
\end{tabular}

FG - fase gasosa / SMD - modelo de solvente

Com os resultados obtidos pode-se esperar uma reação espontânea com energia de ativação relativamente baixa. Comparando a energia livre de Gibbs com as reações realizadas para a hidroxila, tem-se os dados termodinâmicos apresentados na Tabela 2 e cinéticos na Tabela 3:

Tabela 2. Dados termodinâmicos para as duas reações (kcal/mol):

\begin{tabular}{|c|c|c|}
\hline & $\Delta \mathrm{G}(\mathrm{R}=\mathrm{OH})$ & $\Delta \mathrm{G}(\mathrm{R}=\mathrm{Cl})$ \\
\hline $\mathrm{FG}-$ Isolados & $-0,04$ & $-1,16$ \\
\hline $\mathrm{FG}-$ Intermediários & 6,44 & $-2,32$ \\
\hline
\end{tabular}

Tabela 3. Dados cinéticos para as duas reações $(\mathrm{kcal} / \mathrm{mol})$

\begin{tabular}{|c|c|c|}
\hline & $\Delta \mathrm{G}^{\ddagger}(\mathrm{R}=\mathrm{OH})$ & $\Delta \mathrm{G}^{\ddagger}(\mathrm{R}=\mathrm{Cl})$ \\
\hline $\mathrm{FG}-$ Isolados & 66,89 & 29,70 \\
\hline $\mathrm{FG}-$ Intermediários & 73,30 & 27,27 \\
\hline
\end{tabular}

A reação referente ao Modelo 2 mostrouse possivelmente espontânea, tanto para a reação considerando as moléculas isoladas e intermediários, o que não é visto para a reação referente ao Modelo 1. Isso pode ser explicado pelo fato de que o cloro é um grupo abandonador melhor do que a hidroxila, já que o cloro comporta melhor a carga negativa (mais esférico). Observa-se também uma energia de ativação relativamente baixa para o Modelo 2 e alta para o Modelo 1, pode-se afirmar, portanto, que a reação é cineticamente favorável somente para o Modelo 2.

Os resultados experimentais por espectroscopia de absorção na região do infravermelho (FTIR-ATR) indicaram que a formação do cloreto do ácido e sua posterior esterificação com o butan-1,2,diol foram viáveis através da metodologia utilizada. As principais evidências são as variações nos estiramentos $\mathrm{C}=\mathrm{O}\left(V_{\mathrm{C}=\mathrm{O}}\right.$ Ad-COOH 1687, Ad-COCl 1785 e Ad-COOR 1724 cm- $^{-}$ 1). Para o cloreto do ácido é observado o estiramento $V_{\mathrm{C}}$. ${ }_{\mathrm{Cl}}$ em $749 \mathrm{~cm}^{-1}$ e para o produto da esterificação com o butan-1,2, diol são verificados os $V_{\mathrm{C}-\mathrm{O}}$ em 1224 e o $V_{\mathrm{C}-\mathrm{O}-\mathrm{C}}$ em $1070 \mathrm{~cm}^{-1}$. Mais ainda, não são observados os $V_{\mathrm{OH}}$ em 3600 e $1049 \mathrm{~cm}^{-1}$ referentes ao diol, o que sugere a esterificação nas duas hidroxilas, primária e secundária, Figura 6.

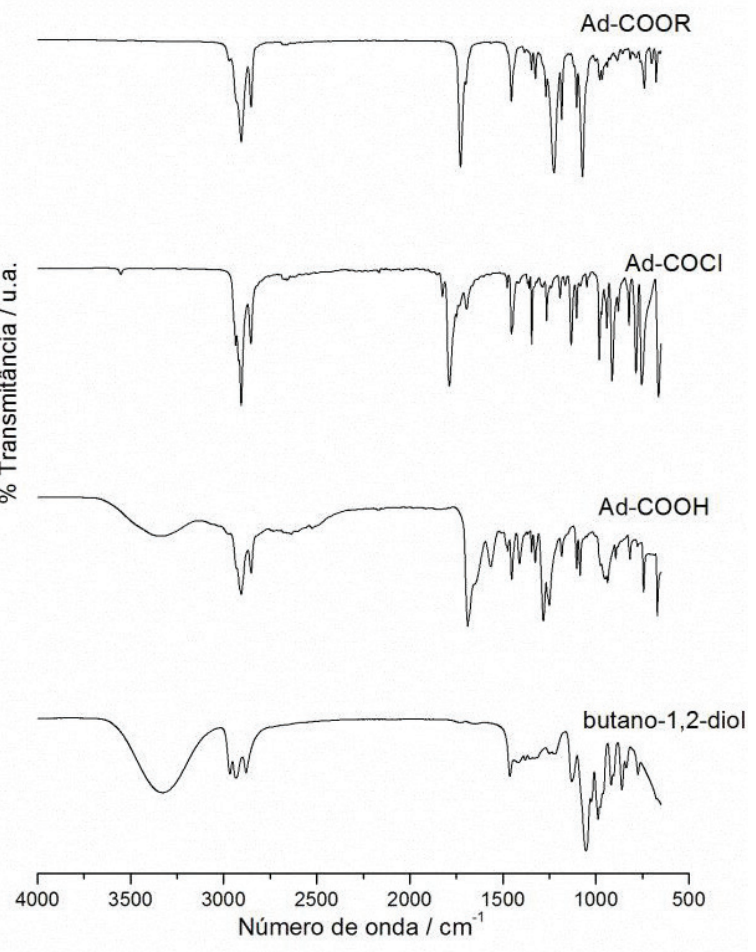

Figura 6. FTIR-ATR dos reagentes utilizados e produto obtido. 
Realizando os cálculos para a segunda reação de esterificação (Figura 2), sendo $\mathrm{R}=\mathrm{Cl}$, foi possível encontrar o estado de transição, apresentado na Figura 7, e seu respectivo gráfico de IRC na Figura 8.

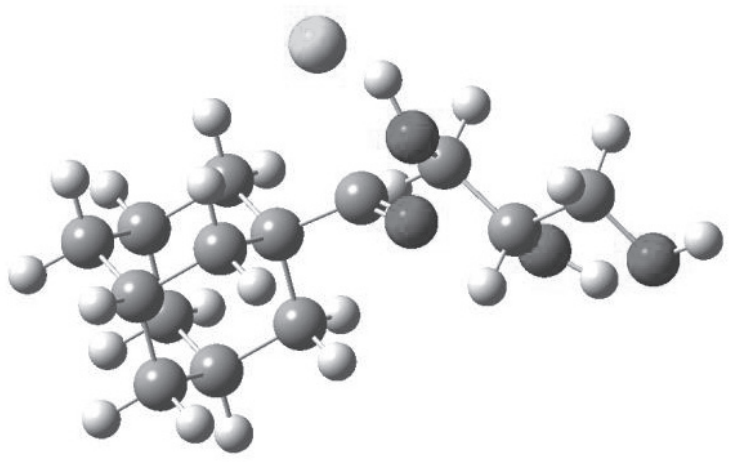

Figura 7. Estado de transição da segunda reação (M062x/SMD/289,39i)

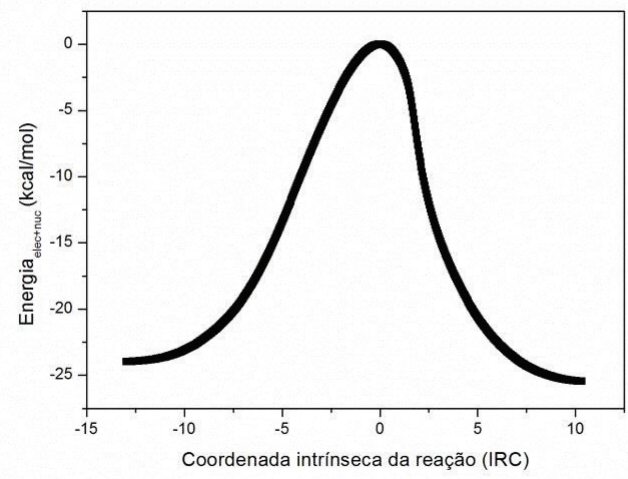

Figura 8. Gráfico da coordenada intrínseca da reação (IRC) para a segunda reação.

Espera-se concluir todos os cálculos para cada um dos modelos descritos, para que se possa realizar o estudo termodinâmico de equilíbrio entre a ciclodextrina e os modelos de adamantano.

\section{Conclusão}

É possível concluir que a reação descrita pelo Modelo 2 tem dados termodinâmicos favoráveis a uma reação espontânea e dados cinéticos relativamente baixos para que a reação ocorra. Comparando os resultados entre os modelos, com o cloro (Modelo 2), têm-se melhores resultados, sendo, portanto preferíveis para os estudos que serão realizados com as ciclodextrinas.

\section{Agradecimentos}

Os autores agradecem o suporte oferecido pela CNPq, FAPEMIG e RQ-MG.

1. D. Thassu, et al. Nanoparticulate Drug Delivery Systems. $1^{\mathrm{a}}$ ed. New York: Informa HealthCare, 2007.

2. F. B. De Sousa, et al. Phys. Chem. Chem. Phys. 14, 1934, (2012).

3. D. Harries, et al. J. Am. Chem. Soc., 127, 2184, (2005).

4. T. Liu, et al. ACS Macro Lett., 4, 357, (2015).

\section{Selma Fabiana Bazan', Clara H. S. Braga ${ }^{2}$, Frederico B. De Sousa ${ }^{2} \&$ Juliana Fedoce Lopes ${ }^{\prime}$}

\footnotetext{
${ }^{1}$ Universidade Federal de Itajubá, LaQC - Laboratório de Química Computacional, Av. BPS nº1303, Bairro: Pinheirinho, 37500-903, Itajubá, MG, Brasil;

${ }^{2}$ Universidade Federal de Itajubá, LSPS - Laboratório de Sistemas Poliméricos e Supramoleculares, Av. BPS nº1303, Bairro: Pinheirinho, 37500-903, Itajubá, MG, Brasil

*E-mail: sfbazan@hotmail.com -
} 